\title{
Grape seed proanthocyanidin extract ameliorates murine autoimmune arthritis through regulation of TLR4/MyD88/NF-кB signaling pathway
}

\author{
Sang-Hyon Kim ${ }^{1}$, Jihye Bang ${ }^{2}$, Chang-Nam Son ${ }^{1}$, Won-Ki Baek ${ }^{3}$, and Ji-Min Kim ${ }^{1}$
}

${ }^{1}$ Division of Rheumatology, Department of Internal Medicine, Keimyung University Dongsan Medical Center, Daegu;

${ }^{2}$ Keimyung University School of Medicine, Daegu; ${ }^{3}$ Department of Microbiology, Keimyung University School of Medicine, Daegu, Korea

Received: March 4, 2016

Revised : April 8, 2016

Accepted: April 27, 2016

\section{Correspondence to}

Ji-Min Kim, M.D.

Division of Rheumatology, Department of Internal Medicine, Keimyung University Dongsan Medical Center, 56 Dalseong-ro, Jung-gu, Daegu 41931, Korea

Tel: +82-53-250-7410

Fax: +82-53-250-7434

E-mail: okjimin@dsmc.or.kr
Background/Aims: Grape seed proanthocyanidin extract (GSPE) has been reported to have a beneficial effect on regulating inflammation. However, the anti-inflammatory mechanism of GSPE remains unclear. The aim of this study was to verify the influence of GSPE on the Toll-like receptor 4 (TLR4)-mediated signaling pathway in the regulation of murine autoimmune arthritis.

Methods: Collagen-induced arthritis (CIA) was induced in dilute brown non-agouti (DBA)/1J mice. The mice were treated with GSPE (o or $100 \mathrm{mg} / \mathrm{kg}$ ) intraperitoneally. The severity of arthritis was assessed clinically, biochemically, and histologically. Immunostaining for TLR4 was performed. The expressions of TLR4 and downstream signaling molecules were analyzed by Western blot. The effect of GSPE on lipopolysaccharide (LPS)-induced TLR4 activation was also evaluated using RAW264.7 cells and fibroblast-like synoviocytes (FLSs) from patients with rheumatoid arthritis and from those with osteoarthritis.

Results: GSPE attenuated the clinical severity of arthritis and decreased histological damage. GSPE treatment reduced the number of TLR4-stained cells in the synovium of mice with CIA. GSPE also downregulated the expression of TLR4, myeloid differentiation factor 88 (MyD88) and phosphorylated IкB $\alpha$ synovial protein in CIA mice. Concurrently, GSPE inhibited the nuclear translocation of nuclear factor- $\kappa \mathrm{B}(\mathrm{NF}-\kappa \mathrm{B})$ subunits (p65 and $\mathrm{p} 50)$. LPS-induced TLR4 activation was suppressed by GSPE in human FLS as well as in murine macrophages in vitro.

Conclusions: Our results demonstrated that GSPE ameliorated CIA by regulating the TLR4-MyD88-NF-кB signaling pathway.

Keywords: Grape seed proanthocyanidin extract; Arthritis, experimental; Tolllike receptor 4; Arthritis, rheumatoid

\section{INTRODUCTION}

Rheumatoid arthritis (RA) is a chronic autoimmune disease characterized by persistent inflammation of the joint synovium and synovial hyperplasia. The aberrant changes, which occur in the synovium, lead to joint damage and functional disability. Although the pathogenesis of RA has not been clearly identified, it is clear that a cascading network of pro-inflammatory cytokines plays a major role in the development of RA [1]. Toll-like receptor 4 (TLR4) contributes to the induction of pro-inflammatory cytokines by activating innate 
immunity under certain circumstances [2]. To date, several studies have suggested that TLR4 is implicated in the pathogenesis of RA. TLR4 is highly expressed in the inflamed synovium of RA patients [3]. The activation of the TLR4 signaling pathway is involved in persistent inflammation and joint destruction in RA [4]. Associations between TLR4 single nucleotide polymorphisms and RA disease activity have been reported [5].

Grape seed proanthocyanidin extract (GSPE) is a standardized water-ethanol extract derived from red grape seeds in which various antioxidants including catechins and oligomeric proanthocyanidins are accumulated [6]. GSPE is made up of a combination of ingredients with $15 \%(+)$ catechin and (-) epicatechin; 80\% (-) epicatechin 3 -O-gallate, dimers, trimers, tetramers, and their gallates; and $5 \%$ pentamers, hexamers, heptamers, and their gallates [7]. Proanthocyanidins are the most abundant phenolic compounds in grape seeds. They have been reported to have various bio-protective functions such as anti-bacterial, anti-viral, anti-allergic, anti-carcinogenic, anti-thrombotic, and vasodilatory activities [8]. Proanthocyanidins possess more potent anti-oxidative activity than vitamins $\mathrm{C}, \mathrm{E}$, and $\beta$-carotene [9]. Furthermore, the anti-inflammatory properties of proanthocyanidins on experimental inflammation, including in animal models of ulcerative colitis, skin tumors, and collagen-induced arthritis (CIA), have been shown in previous studies [10-13]. However, little information is available concerning how proanthocyanidins regulate experimental autoimmune arthritis. In this study, we hypothesized that GSPE would influence TLR4 signaling pathways, which play a critical role in the pathogenesis of RA, in the development of murine autoimmune arthritis. We also examined the immunological effect of GSPE on fibroblast-like synoviocytes (FLSs) in patients with RA.

\section{METHODS}

\section{Experimental animals}

Dilute brown non-agouti (DBA)/1J mice (6 to 8 weeks old; SLC Inc., Hamamatsu, Japan), were housed in polycarbonate cages and fed with standard mouse chow (Ralston Purina, St. Louis, MO, USA) and water ad libitum. All experimental procedures were examined and approved by the Animal Research Ethics Committee of the Keimyung University (No. KM-2009-06R).

\section{Induction of $\mathrm{CIA}$ and GSPE treatment}

Male DBA/1J mice were given an intradermal injection of $100 \mu \mathrm{g}$ of bovine type II collagen emulsified in complete Freund's adjuvant (1:1, w/v; Chondrex, Redmond, WA, USA) into the base of the tail. Three weeks after primary immunization, a booster, which consisted of 100 $\mu \mathrm{g}$ of bovine type II collagen emulsified in incomplete Freund's adjuvant (IFA; 1:1, v/v; Chondrex), was injected intradermally. Mice were divided into three groups; control, CIA, and CIA with GSPE treatment. To investigate the effects of GSPE on the development of arthritis, CIA mice were treated with GSPE (100 mg/kg body weight) five times per week for 3 weeks intraperitoneally, after the booster injection. GSPE was kindly provided by Hanlim Pharmaceutical Company (Seoul, Korea). GSPE dissolved in saline was used for the experiments.

\section{Clinical arthritis severity score}

Three observers scored the severity of arthritis independently from day 24 after primary immunization to day 42. Clinical arthritis scores of the mice were evaluated as follows: o, no evidence of swelling; 1, mild swelling confined to the ankle or the midfoot (tarsals); 2, moderate swelling extending from the ankle to the midfoot (tarsals); and 3, severe swelling and erythema extending from the ankle to the metatarsal joints or ankylosis of the ankle joint. The sum of the scores of four limbs represented the arthritis score of each mouse.

\section{Histological analysis}

Joint tissues were fixed with $4 \%$ paraformaldehyde. They were then decalcified for 1 week in hydrochloric acid before being embedded in paraffin. Tissue sections ( $4 \mu \mathrm{m})$ were stained with H\&E in order to determine the influx of inflammatory cells. Inflammation was scored using the following criteria: o, no inflammation; 1 , slight thickening of lining layer or some infiltrating cells in sublining layer; 2, slight thickening of lining layer plus some infiltrating cells in sublining layer; 3 , thickening of lining layer, influx of cells in sublining layer and presence of cells in the synovial space and synovium highly infiltrated with many inflammatory cells. The tissue sections were stained with safranin $\mathrm{O}$ to evaluate carti- 
lage degradation under light microscopic examination. The extent of cartilage damage scored using the following criteria: o, no destruction; 1 , minimal erosion limited to single spots; 2 , slight to moderate erosion in a limited area; 3, more extensive erosion and destruction. Immunohistochemistry was performed using the Vectastain $\mathrm{ABC}$ kit and 3,3'-diaminobenzidine as a substrate (both from Vector Laboratories, Burlingame, CA, USA). Nonspecific staining was reduced by protein blocking with $20 \%$ normal goat serum for 30 minutes, at room temperature. Joint tissues were incubated with anti-TLR4 antibody (Santa Cruz Biotechnology, Dallas, TX, USA) overnight at $4^{\circ} \mathrm{C}$.

\section{Enzyme-linked immunosorbent assay}

The serum levels of anti-type II collagen specific immunoglobulin G (IgG; Chondrex), tumor necrosis factor $\alpha$ (TNF- $\alpha$ ), interleukin 6 (IL-6), and IL-17 (R\&D Systems, Minneapolis, MN, USA) were measured according to the manufacturer's protocol using an enzyme-linked immunosorbent assay (ELISA) kit (R\&D). For the determination of cytokine levels, blood samples from the mice were obtained on day 42 and stored at $-70^{\circ} \mathrm{C}$ until use. Antibody concentrations for each serum sample were obtained by reference to the standard curve. The absorbance values were determined at $450 \mathrm{~nm}$ with an ELISA microplate reader (Vector3, Perkin Elmer, Waltham, MA, USA).

\section{Protein extraction and Western blot analysis}

Ankles joints of mice were isolated by disarticulation distal to tibia. Synovial tissues from hind paws were collected and frozen until experiments. The frozen synovial tissues were pulverized and synovial protein was extracted as follows. Pulverized synovial tissues were lysed in ice-cold lysis buffer ( $\mathrm{pH} 7.4,50 \mathrm{mM}$ Tris-HCl, $1 \%$ Nonidet P-40, $0.25 \%$ sodium deoxycholate, $150 \mathrm{mM} \mathrm{NaCl}, 1$ $\mathrm{mM} \mathrm{Na}_{3} \mathrm{VO}_{4}$, and $1 \mathrm{mM} \mathrm{NaF}$ ) containing protease inhibitors (2 mM phenylmethylsulfonyl fluoride, $100 \mu \mathrm{g} / \mathrm{mL}$ leupeptin, $10 \mu \mathrm{g} / \mathrm{mL}$ pepstatin, $1 \mu \mathrm{g} / \mathrm{mL}$ aprotinin, and 2 $\mathrm{mM}$ ethylenediaminetetraacetic acid). Cytoplasmic and nuclear extracts were prepared from joint tissues using the NE-PER nuclear and cytoplasmic protein extraction reagent kit (Thermo Pierce, Rockford, IL, USA). Samples in each group were subjected to $10 \%$ sodium dodecyl sulfate polyacrylamide gel electrophoresis on resolving gels appropriately and transferred to a nitrocellulose membrane (Millipore, Billerica, MA, USA). Blots were incubated overnight at $4^{\circ} \mathrm{C}$ with primary antibodies for TLR4, myeloid differentiation factor 88 (MyD88), Toll/IL-1 receptor domain-containing adaptor induc-

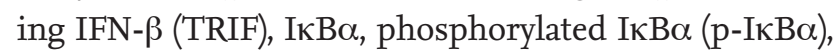
p65, p5o (all from Santa Cruz Biotechnology), and $\beta$-actin (Sigma-Aldrich, St. Louis, MO, USA). Anti-laminB1 (Santa Cruz Biotechnology) was used as an internal control of nucleus protein marker. The membrane was then washed with a mixture of Tris-buffered saline with Tween 20 (TBS-T) and incubated with horseradish peroxidase-conjugated secondary antibodies (Santa Cruz Biotechnology). Protein bands were detected using enhanced chemiluminescent reagents (Amersham Pharmacia Biotech, Uppsala, Sweden).

\section{Isolation and culture of FLS}

FLS were isolated by enzymatic digestion of synovial tissue, provided by the Keimyung Human Bio-Resource Bank, a member of the National Biobank of Korea. The tissue was obtained from patients with osteoarthritis (OA) and from those with RA who underwent total joint replacement surgery or a synovectomy at Keimyung University Dongsan Medical Center. All the patients fulfilled the American College of Rheumatology criteria and agreed to give their informed consent (approved by the Institutional Review Board [IRB] of Keimyung University Dongsan Medical Center [IRB No. 2015-02005]) [14]. The tissue samples were minced into 2 to 3 $\mathrm{mm}$ pieces and treated for 4 hours with $0.5 \mathrm{mg} / \mathrm{mL}$ type II collagenase (Worthington Biochemical Corp., Lakewood, NJ, USA) in Dulbecco's modified Eagle's medium (DMEM) at $37^{\circ} \mathrm{C}$ in a water bath. Synoviocytes were grown in DMEM supplemented with 10\% fetal bovine serum, $2 \mathrm{mM}$ L-glutamine, 100 units/mL penicillin, and $100 \mathrm{ng} / \mathrm{mL}$ streptomycin. The cells were used for experiments from passage 4 to 8 , at which time the population was homogeneous.

\section{Statistical analysis}

The statistical analysis was performed using the SPSS version 22 (IBM Co., Armonk, NY, USA). Student $t$ tests and one-way analysis of variance were carried out. The accepted level of significance was preset at $p<0.05$. Each experiment was executed at least three times in duplicate. Data are presented as mean $\pm \mathrm{SD}$. 


\section{RESULTS}

\section{GSPE ameliorates murine autoimmune arthritis}

We examined the effect of GSPE on the development of experimental arthritis in vivo using a CIA mouse model. $\mathrm{DBA} / \mathrm{1J}$ mice were intraperitoneally injected with GSPE five times per week for 3 weeks after the second immunization with type II collagen/IFA. The mean arthritis score of GSPE-treated CIA mice was significantly lower than that of CIA mice (Fig. 1A). Representative histologic sections of hind paws of the mice are shown in Fig. 1B. The joints of CIA mice revealed the infiltration of inflammatory cells, synovial proliferation, and cartilage destruction (Fig. 1B). In CIA mice treated with GSPE, there was a reduction in infiltrated inflammatory cells, synovial proliferation, and cartilage damage (Fig. 1B).

\section{GSPE decreases serum levels of pro-inflammatory cytokines in CIA mice}

We investigated whether the therapeutic effect of GSPE in CIA mice affects the humoral immune response to type II collagen and serum levels of pro-inflammatory cytokines. The levels of anti-type II collagen IgG, TNF- $\alpha$, IL-6, and IL-17 in serum samples of mice were measured using ELISA, 6 weeks after primary immunization. The serum concentrations of anti-type II collagen IgG were significantly reduced in CIA mice following GSPE treatment, when compared with CIA mice (Fig. 2A). There was also a decrease in serum levels of TNF- $\alpha$ (Fig. $2 \mathrm{~B}$ ), IL-6 (Fig. 2C), and IL-17 (Fig. 2D) in the GSPE-treated group.

\section{GSPE administration reduces TLR4 expression in the synovium of CIA mice}

TLR4 has been reported to play a critical role in the pathogenesis of CIA $[15,16]$. Therefore, we investigated whether GSPE treatment influenced TLR4 expression in the synovial tissue of CIA using immunohistochemical staining. To assess the in vivo effect of GSPE on TLR4 expression, histological sections of the tibiotalar joints were stained in order to visualize the presence of TLR4. As shown in Fig. 3A, less TLR4-positive cells were observed in the joints of GSPE-treated CIA mice than in those of CIA mice. We also investigated the ef-
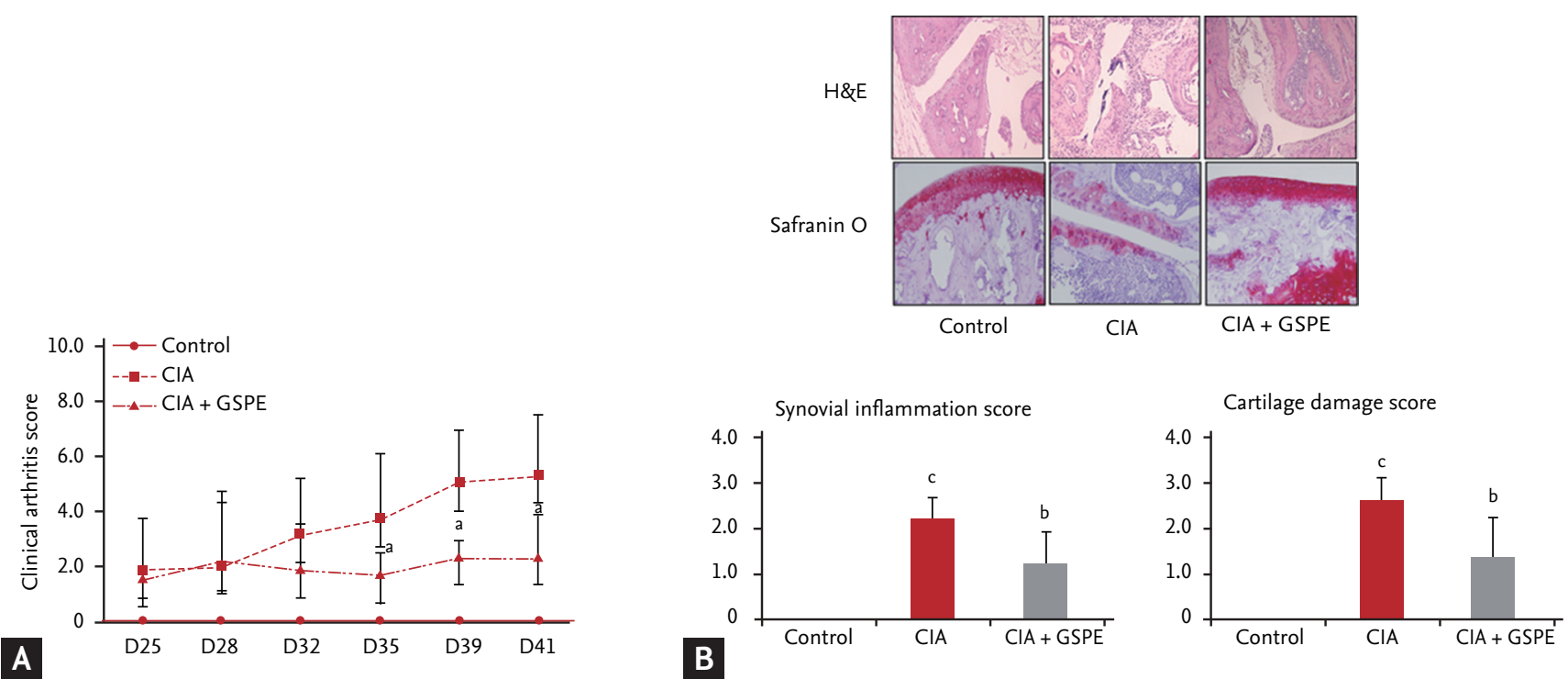

Figure 1. Grape seed proanthocyanidin extract (GSPE) treatment relieves the severity of collagen-induced arthritis (CIA) in mice ( $\mathrm{n}=8$ for each group; control, CIA, GSPE-treated CIA group). (A) For induction of CIA, male dilute brown non-agouti (DBA)/1J mice were given $100 \mu \mathrm{g}$ of bovine type II collagen emulsified in complete Freund's adjuvant intradermally into the base of the tail. A booster injection was administered 3 weeks later. GSPE (100 mg/kg) was initiated intraperitoneally after the booster injection. The mice were treated with GSPE (100 mg/kg) five times per week for 3 weeks. The arthritis score for each mouse was calculated as the sum of the scores of both hind paws. Mean values of the arthritis scores are represented on the graph. Error bars indicate standard deviation of the mean. (B) A hind limb of each mouse was stained with H\&E (upper panel) and safranin O (lower panel). The joints from CIA mice treated with GSPE demonstrated attenuated inflammation and cartilage destruction when compared with those from CIA mice $(\times 200)$. Error bars indicate standard deviation of the mean. ${ }^{\mathrm{a}} \mathrm{p}<0.05$, ${ }^{b} p<0.01$, compared with the CIA group, ${ }^{c} p<0.001$ compared with the control group. 


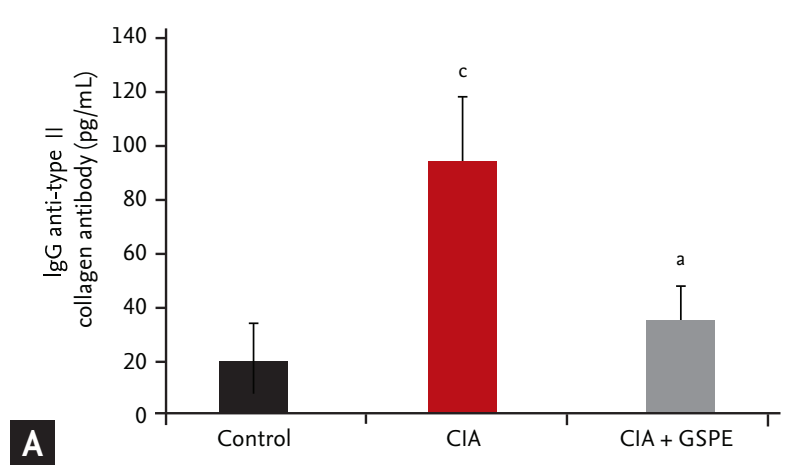

C

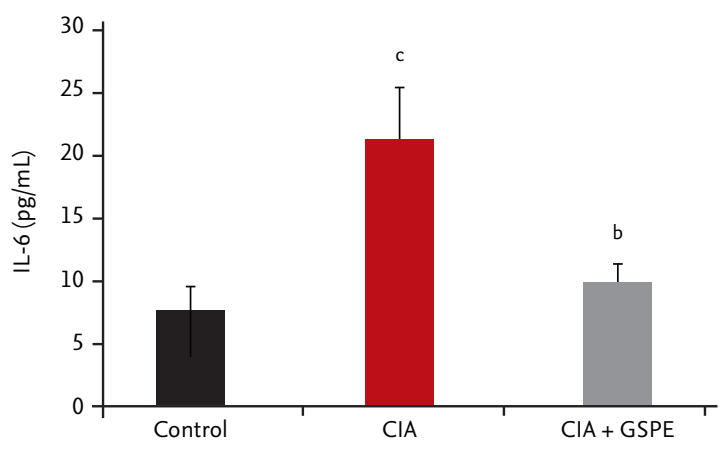

B

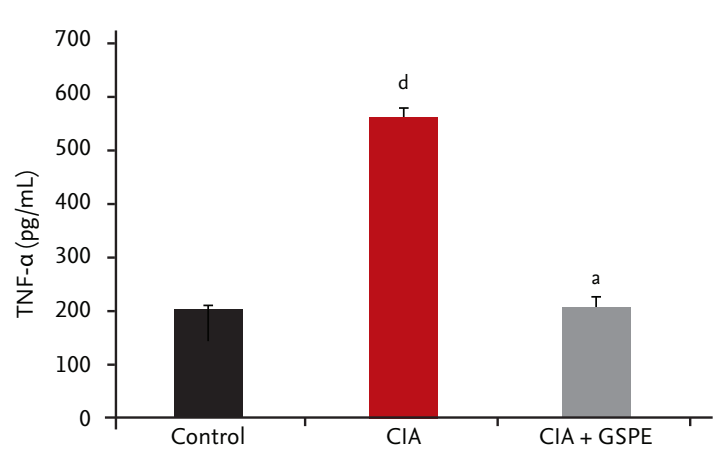

D

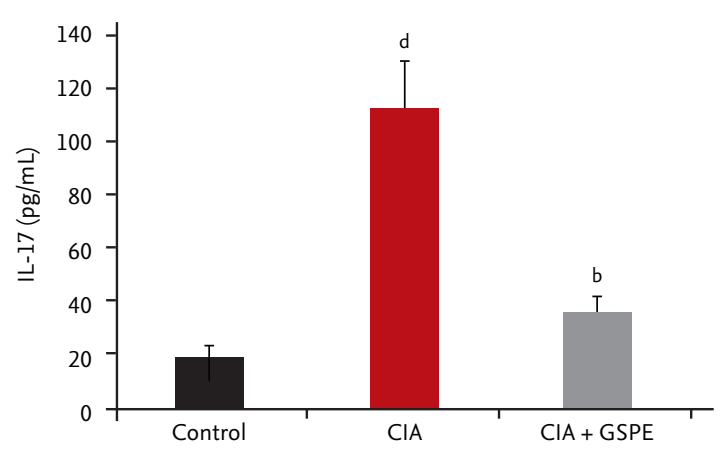

Figure 2. Grape seed proanthocyanidin extract (GSPE) treatment suppresses immune response to type II collagen and decreases serum levels of pro-inflammatory cytokines. Serum concentrations of (A) type II collagen specific total immunoglobulin G (IgG), (B) tumor necrosis factor $\alpha$ (TNF- $\alpha$ ), (C) interleukin 6 (IL-6), and (D) IL-17 were determined using enzyme-linked immunosorbent assay in each group of mice ( $\mathrm{n}=8$ for each group). Error bars represent standard deviation of the mean. Each experiment was performed three times. ${ }^{a} p<0.05,{ }^{b} p<0.01$ compared with the collagen-induced arthritis (CIA) group, ${ }^{c} p<0.05,{ }^{d} p<$ 0.001 compared with the control group.

fect of GSPE on the intracellular proteins involved in TLR4-mediated signal transduction in the synovium of CIA, by using Western blotting. Synovial protein levels of TLR4, MyD88, and TRIF were analyzed. The expression levels of TLR4 and MyD88 proteins were upregulated in the synovial extracts of CIA mice when compared with those of control mice. TLR4 and MyD88 expression levels were downregulated in GSPE-treated CIA mice when compared with CIA mice (Fig. 3B). The expression level of TRIF showed no increase in CIA mice when compared to the control mice. However, GSPE treatment downregulated the expression level of TRIF in CIA mice when compared to CIA mice (Fig. 3B). In order to explore the effects of GSPE on the activation of

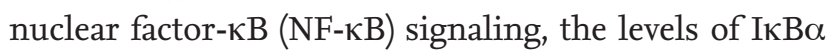
and $\mathrm{p}-\mathrm{I} \kappa \mathrm{B} \alpha$ were measured. GSPE reduced the level of $\mathrm{p}-\mathrm{I} \kappa \mathrm{B} \alpha$ protein in the synovial tissue of CIA mice (Fig.

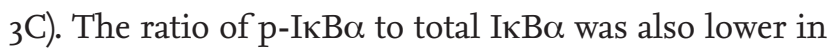
GSPE-treated CIA mice than in CIA mice (Fig. ${ }_{3} \mathrm{C}$ ). We also examined whether GSPE affected nuclear translo- cation of the NF- $\mathrm{kB}$ subunits, p65 and p5o. The nuclear extracts and cytoplasmic lysates were purified from the synovial tissue and subjected to Western blotting. The nuclear localization of p65 and p5o NF- $\mathrm{kB}$ was inhibited in the synovial extracts of GSPE-treated CIA mice, while nuclear localization of $\mathrm{p} 65$ and $\mathrm{p} 50 \mathrm{NF}-\mathrm{\kappa B}$ was prominent in those of CIA mice (Fig. 3D).

\section{GSPE suppresses lipopolysaccharide-induced TLR4 activation in RAW264.7 cells}

Our data demonstrates that GSPE administration decreased TLR4 expression in the synovium of CIA mice (Fig. 3A and 3B). We next investigated whether GSPE had an influence on TLR4 expression in vitro by using a murine macrophage cell line, RAW264.7. GSPE downregulated the expression of TLR4, which was enhanced by stimulation with lipopolysaccharide (LPS) in RAW264.7 cells (Fig. 4A). We also evaluated the effect of GSPE on the expression of TLR4 in FLS from patients with OA and RA. As shown in Fig. 4B, LPS-induced TLR4 upreg- 

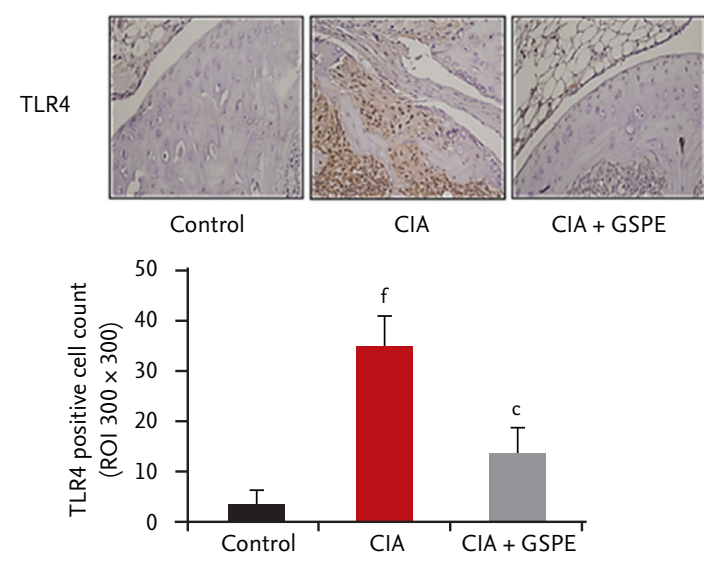

A
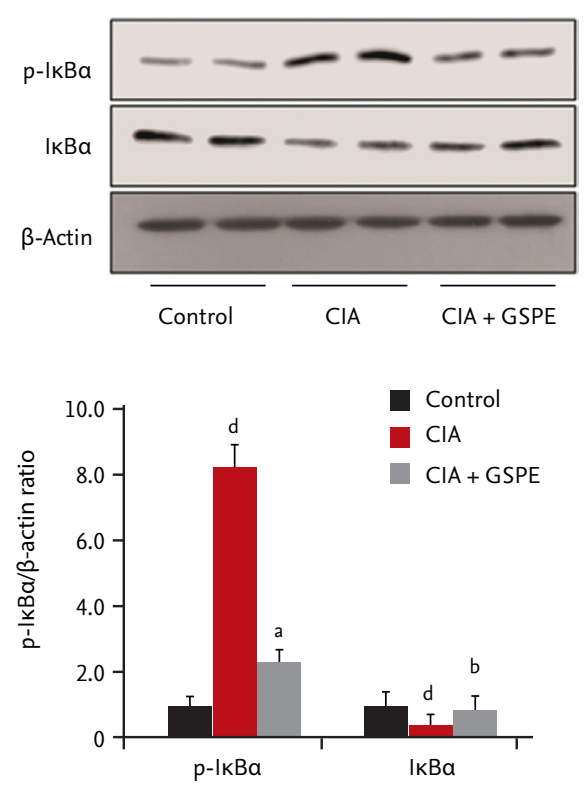

C

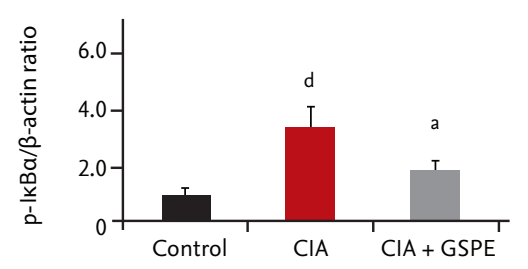

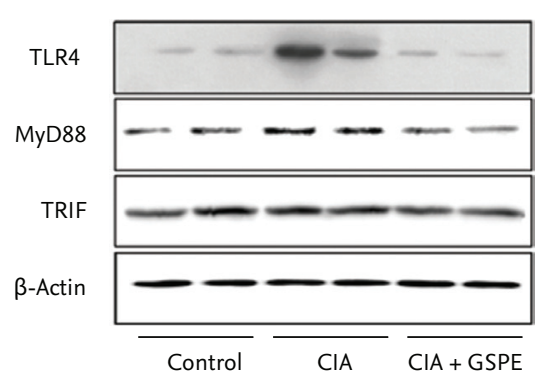

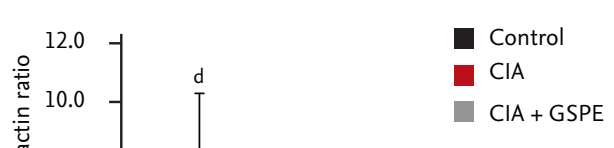

B
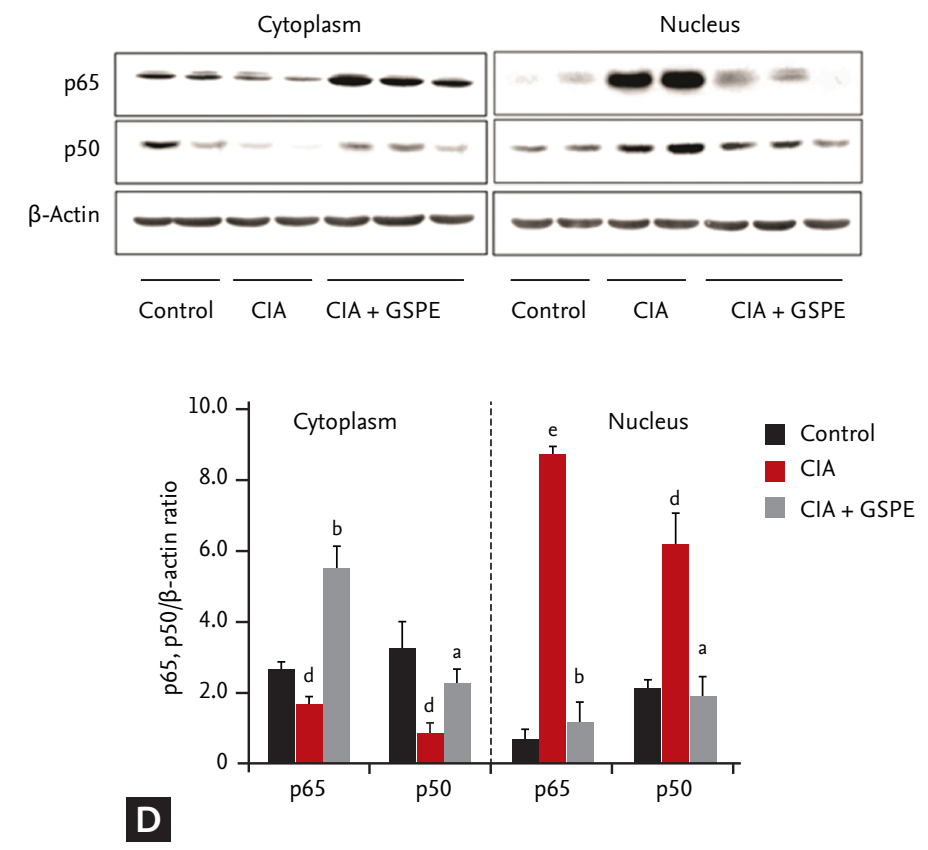

Figure 3. Grape seed proanthocyanidin extract (GSPE) downregulates Toll-like receptor 4 (TLR4) expression and TLR4-mediated signal transduction proteins in the synovium of mice with collagen-induced arthritis (CIA; $n=8$ for each group). (A) TLR4 expression was assessed by immunohistochemical staining. GSPE treatment led to a reduction in TLR4-positive cells in the synovium of CIA mice, whereas the number of TLR4-stained cells was markedly increased in the synovium of CIA mice, when compared to that of control mice ( $\times 200)$. (B) Western blot analysis of synovial extracts from GSPE-treated CIA mice showed a decreased protein level of TLR4, myeloid differentiation factor 88 (MyD88), and Toll/interleukin 1 receptor domain-containing adaptor inducing IFN- $\beta$ (TRIF), when compared with CIA mice. (C) The expression level of phosphorylated IкB $\alpha$ (p-IкB $\alpha$ ) was also reduced in GSPE-treated CIA mice. (D) GSPE suppressed nuclear translocation of nuclear factor- $\kappa$ B subunits (p65 and p50) in the synovium of CIA mice. Error bars reflect standard deviation of the mean. Each experiment was performed three times. ROI, region of interest. ${ }^{a} p<0.05,{ }^{b} p<0.01,{ }^{c} p<0.001$ compared with the CIA group, ${ }^{d} p<0.05,{ }^{e} p<0.01,{ }^{f} p<0.001$ compared with the control group. 

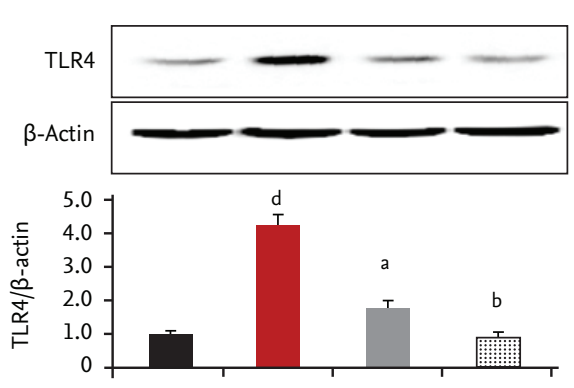

LPS

A
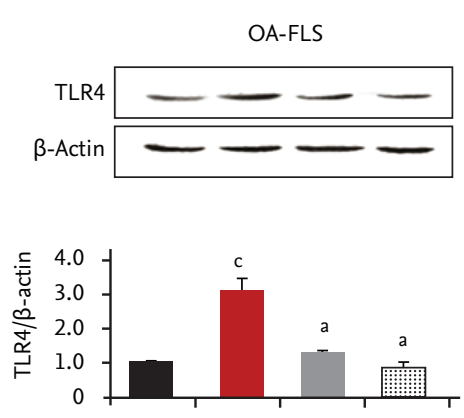

LPS
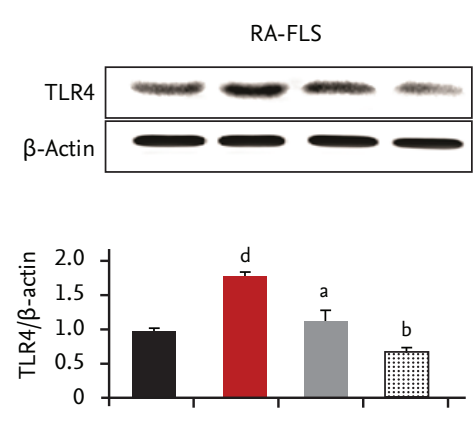

B

GSPE - $\quad-\quad+\quad+$

Figure 4. Grape seed proanthocyanidin extract (GSPE) inhibits lipopolysaccharide (LPS)-induced Toll-like receptor 4 (TLR4) expression in vitro. (A) RAW264.7 macrophages were co-incubated with or without LPS (1 $\mu \mathrm{g} / \mathrm{mL})$ for 8 hours after pretreatment with or without GSPE $(25 \mu \mathrm{g} / \mathrm{mL})$ for 16 hours. LPS-induced TLR4 expression was downregulated in RAW264.7 cells after treatment with GSPE. (B) Fibroblast-like synoviocyte (FLS) from patients with osteoarthritis (OA; $n=5)$ and rheumatoid arthritis (RA; $\mathrm{n}=3$ ) were incubated in the same conditions as panel A. GSPE treatment reduced LPS-induced TLR4 expression in FLS from patients with RA as well as in those from patients with OA. Data are presented as the mean \pm SD of three independent experiments. Each experiment was performed three times. ${ }^{a} p<0.05,{ }^{b} p<0.01$ compared with the LPS only-stimulated group, ${ }^{c} p<0.05$, ${ }^{d} p<0.01$ compared with the nil (control condition).

ulation was suppressed by GSPE in FLS from patients with RA and OA.

\section{DISCUSSION}

Proanthocyanidins, which are the main constituents of GSPE, belong to the category of condensed tannins [17]. The sources of proanthocyanidins include fruits, vegetables, nuts, seeds, flowers, and bark [18]. Proanthocyanidins are well-known as naturally occurring anti-oxidants. They have been reported to have beneficial effects on modulating inflammation in human cells, such as differentiated adipocytes and human pulmonary epithelial cells in vitro, in addition to animal inflammatory models as mentioned above [19,20]. Some previous studies have shown that GSPE has an anti-inflammatory effect in an animal model of RA. Cho et al. [13] first reported about the therapeutic effect of GSPE on CIA and showed the effects of GSPE on oxidative stress and osteoclastogenesis in vitro. Another study using a murine model of RA has demonstrated the effect of GSPE focusing on inflammation-associated bone destruction [21]. GSPE attenuated both arthritis and obesity in obese CIA mice [22]. However, little is known about the mechanism by which GSPE regulate the inflammatory response. Oxidative stress is reduced by GSPE, and has been reported to be involved in the pathogenesis of murine autoimmune arthritis. However, there has been controversy as to whether oxidative stress instigates, or suppresses the inflammation in a model of murine autoimmune arthritis [23-25]. One previous study reported that GSPE influences murine autoimmune arthritis by regulating Foxp $^{+}$regulatory and IL-17-producing T cells, reciprocal control of which is important in managing autoimmune arthritis [26]. There was another report that GSPE has an anti-arthritic effect in adjuvant-induced arthritis model by modifying $\mathrm{T}$ cell subsets [27]. In this study, we focused on revealing the mechanism of action of GSPE in suppressing murine autoimmune arthritis. Here, we demonstrated that GSPE has an anti-arthritic effect through the regulation of TLR4-mediated signaling for the first time.

TLRs are innate receptors, which play an essential role in the innate immune system [28]. Among diverse TLRs, TLR4 was the first to be characterized, and has been reported to play a significant role in the pathogenesis of autoimmune diseases [29]. In particular, a large amount of research provides evidence that TLR4 is implicated in the pathogenesis of RA. TLR4-deficient mice showed lower degree of severity and incidence of CIA compared to the wild-type mice [15]. LPS-induced TLR4 activation was greatly enhanced in peripheral blood mononuclear cells (PBMCs) from patients with RA than in those from 
patients with OA or healthy controls [30]. TLR4 activation by LPS induces the production of pro-inflammatory cytokines in human FLS and PBMCs from patients with RA [31,32]. TLR4 upregulation increases pro-inflammatory activity and induces degeneration in chondrocytes [33]. Although controversy exists regarding the association between TLR4 polymorphisms and RA, previous studies have reported that a functional variant of TLR4 is associated with susceptibility to RA and that non-missense genetic polymorphisms of TLR4 confer the risk of the development of RA [34,35]. Our data demonstrated that TLR4 is highly expressed in the joints of CIA mice. This observation supports a previous study, which confirmed that treatment with a TLR4 antagonist decreased the severity of CIA [16]. In this study, GSPE-treated mice had fewer TLR4-expressing cells in their synovium in comparison to those that were not treated with GSPE. This suggests that GSPE inhibits TLR4 expression in the synovium in vivo. However, little is known about the effects of GSPE on the expression of TLR4 in the synovium of CIA mice. Therefore, further investigation into the mechanism by which GSPE suppresses the expression of TLR4 in the synovium is required.

TLR4 is regarded as an important receptor in inflammatory responses. TLR4 ligation leads to the activation of a downstream transcription factor, NF- $\kappa \mathrm{B}$, which is linked by MyD88, a key adaptor protein in TLR4-NF- $\mathrm{B}$ signaling. The stimulation of TLR4 recruits the MyD88 adaptor protein, leading to IKB kinase activation and

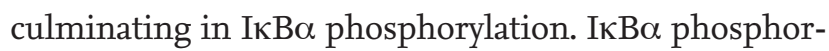
ylation releases cytosolic sequestration of p65 and p5o NF-кB subunits and causes nuclear repositioning of those proteins. In this study, we evaluated the in vivo effect of GSPE on NF- $\mathrm{BB}$ and MyD88. We also examined the influence of GSPE on the various steps in NF-кB activation by performing cell fractionation experiments. Our data showed that GSPE treatment diminishes the phosphorylation of I $\mathrm{B} \alpha$ and inhibits nuclear translocation of p65 and p5o NF- $\mathrm{kB}$ subunits. The results indicate that GSPE inhibits NF- $\kappa B$ activation through the suppression of $\mathrm{I} \kappa \mathrm{B} \alpha$ phosphorylation. In addition to MyD88, TRIF is also an adaptor protein, which responds to the activation of TLR4 [36]. However, the data presented here show no significant change in the expression of TRIF between CIA and control mice. These results may imply that the inflammatory responses of CIA uti- lize the TLR4-MyD88-dependent pathway rather than TLR4-TRIF-dependent pathway, although further studies are required to confirm these tentative implications.

Prior to in vitro experiments detailed above, we also evaluated the cytotoxic effect of GSPE on FLS from RA or OA patients, and RAW264.7 cells using the 3-(4,5-dimethylthiazol-2-yl)-2,5-diphenylphenyltetrazolium bromide(MT'T) assay. After a 24-hour incubation period, GSPE, at concentrations between o and $50 \mu \mathrm{g} / \mathrm{mL}$ showed no evidence of cytotoxicity to both FLS and RAW264.7 cells (data not shown). FLS in the synovium play a critical role in maintaining inflammation and destroying cartilage by producing pro-inflammatory cytokines [37]. FLS have been reported to be able to express TLR4 as well as a variety of adhesion molecules [37,38]. Macrophages, which are the main source of pro-inflammatory cytokines such as TNF- $\alpha$ and IL-1, also participate in inducing inflammation and bone erosion in patients with RA [39]. The in vitro data from the present study demonstrated that GSPE also has an influence on TLR4 expression in human cells in addition to murine cells.

This study has some limitation that the study was designed to include only three groups; control group, GSPE-untreated CIA group, and GSPE-treated CIA group. Inclusion of GSPE-treated control group would have given more information about basic effect of GSPE on control DBA/1J mice. We chose one dosage (100 mg/ $\mathrm{kg}$ ) of GSPE in the study based on previous study as our study was focused on revealing the new mechanism of action of GSPE [13]. However, different dosage group of GSPE would have made the study more informative. CIA group co-treated with GSPE and TLR4 agonist would have also provided more information that TLR4 inhibition by GSPE could be reversed by TLR4 agonist in CIA mice. GSPE was given intraperitoneally as we intended to administer more accurate dosage of GSPE to each mouse in our study. However, oral administration of GSPE might approach to clinical applications more closely.

In conclusion, our study demonstrated that GSPE attenuated autoimmune arthritis by regulating the TLR4/ MyD88/NF-кB signaling pathway. GSPE effectively suppressed anti-type II collagen IgG and pro-inflammatory cytokines. A crucial innate receptor for autoimmune diseases, TLR4 expression in the synovial tissue was significantly influenced by GSPE in vivo, as well as in vitro. 
The results suggest that GSPE could be effective in treating immunological diseases such as RA, in the pathogenesis of which TLR4 activation is involved.

\section{KEY MESSAGE}

1. Our study demonstrated that grape seed proanthocyanidin extract (GSPE) ameliorated collagen-induced arthritis by regulating the Toll-

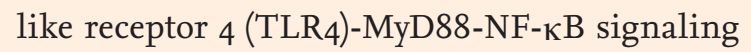
pathway.

2. GSPE also regulated the expression of TLR4 in human fibroblast-like synoviocytes.

3. Further studies would provide more evidence that GSPE could be effective in treating immunological diseases such as rheumatoid arthritis, in the pathogenesis of which TLR4 activation is involved.

\section{Conflict of interest}

No potential conflict of interest relevant to this article was reported.

\section{Acknowledgments}

This work was supported by the research promoting grant from the Keimyung University Dongsan Medical Center in 2013.

\section{REFERENCES}

1. Annunziato F, Cosmi L, Liotta F, Maggi E, Romagnani S. Type $17 \mathrm{~T}$ helper cells-origins, features and possible roles in rheumatic disease. Nat Rev Rheumatol 2009;5:325-331.

2. Pasare C, Medzhitov R. Toll-like receptors: linking innate and adaptive immunity. Adv Exp Med Biol 2005;560:11-18.

3. Radstake TR, Roelofs MF, Jenniskens YM, et al. Expression of toll-like receptors 2 and 4 in rheumatoid synovial tissue and regulation by proinflammatory cytokines interleukin-12 and interleukin-18 via interferon-gamma. Arthritis Rheum 2004;50:3856-3865.

4. Ospelt C, Brentano F, Rengel Y, et al. Overexpression of toll-like receptors 3 and 4 in synovial tissue from patients with early rheumatoid arthritis: toll-like receptor expression in early and longstanding arthritis. Arthritis Rheum
2008;58:3684-3692.

5. Davis ML, LeVan TD, Yu F, et al. Associations of toll-like receptor (TLR)-4 single nucleotide polymorphisms and rheumatoid arthritis disease progression: an observational cohort study. Int Immunopharmacol 2015;24:346-352.

6. Bagchi D, Bagchi M, Stohs SJ, et al. Free radicals and grape seed proanthocyanidin extract: importance in human health and disease prevention. Toxicology 2000;148:187-197.

7. Gabetta B, Fuzzati N, Griffini A, et al. Characterization of proanthocyanidins from grape seeds. Fitoterapia 2000;71:162-175.

8. Fine AM. Oligomeric proanthocyanidin complexes: history, structure, and phytopharmaceutical applications. Altern Med Rev 2000;5:144-151.

9. Zhang XY, Li WG, Wu YJ, Bai DC, Liu NF. Proanthocyanidin from grape seeds enhances doxorubicin-induced antitumor effect and reverses drug resistance in doxorubicin-resistant K562/DOX cells. Can J Physiol Pharmacol 2005;83:309-318.

10. Li WG, Zhang XY, Wu YJ, Tian X. Anti-inflammatory effect and mechanism of proanthocyanidins from grape seeds. Acta Pharmacol Sin 2001;22:1117-1120.

11. Li XL, Cai YQ, Qin H, Wu YJ. Therapeutic effect and mechanism of proanthocyanidins from grape seeds in rats with TNBS-induced ulcerative colitis. Can J Physiol Pharmacol 2008;86:841-849.

12. Meeran SM, Vaid M, Punathil T, Katiyar SK. Dietary grape seed proanthocyanidins inhibit 12-O-tetradecanoyl phorbol-13-acetate-caused skin tumor promotion in 7,12-dimethylbenz[a]anthracene-initiated mouse skin, which is associated with the inhibition of inflammatory responses. Carcinogenesis 2009;30:520-528.

13. Cho ML, Heo YJ, Park MK, et al. Grape seed proanthocyanidin extract (GSPE) attenuates collagen-induced arthritis. Immunol Lett 2009;124:102-110.

14. Aletaha D, Neogi T, Silman AJ, et al. 2010 Rheumatoid arthritis classification criteria: an American College of Rheumatology/European League Against Rheumatism collaborative initiative. Arthritis Rheum 2010;62:25692581.

15. Pierer M, Wagner U, Rossol M, Ibrahim S. Toll-like receptor 4 is involved in inflammatory and joint destructive pathways in collagen-induced arthritis in DBA1J mice. PLoS One 2011;6:e23539.

16. Abdollahi-Roodsaz S, Joosten LA, Roelofs MF, et al. In- 
hibition of Toll-like receptor 4 breaks the inflammatory loop in autoimmune destructive arthritis. Arthritis Rheum 2007;56:2957-2967.

17. Bravo L. Polyphenols: chemistry, dietary sources, metabolism, and nutritional significance. Nutr Rev 1998;56:317333 .

18. Bagchi D, Garg A, Krohn RL, Bagchi M, Tran MX, Stohs SJ. Oxygen free radical scavenging abilities of vitamins $\mathrm{C}$ and $\mathrm{E}$, and a grape seed proanthocyanidin extract in vitro. Res Commun Mol Pathol Pharmacol 1997;95:179-189.

19. Chacon MR, Ceperuelo-Mallafre V, Maymo-Masip E, et al. Grape-seed procyanidins modulate inflammation on human differentiated adipocytes in vitro. Cytokine 2009;47:137-142.

20. Kim H, Kim JY, Song HS, Park KU, Mun KC, Ha E. Grape seed proanthocyanidin extract inhibits interleukin-17-induced interleukin-6 production via MAPK pathway in human pulmonary epithelial cells. Naunyn Schmiedebergs Arch Pharmacol 2011;383:555-562.

21. Park JS, Park MK, Oh HJ, et al. Grape-seed proanthocyanidin extract as suppressors of bone destruction in inflammatory autoimmune arthritis. PLoS One 2012;7:e51377.

22. Jhun JY, Moon SJ, Yoon BY, et al. Grape seed proanthocyanidin extract-mediated regulation of $\mathrm{STAT}_{3}$ proteins contributes to Treg differentiation and attenuates inflammation in a murine model of obesity-associated arthritis. PLoS One 2013;8:e78843.

23. Choi EM. Oxidative status of DBA/1J mice with type II collagen-induced arthritis. J Appl Toxicol 2007;27:472-481.

24. McCubbin MD, Hou G, Abrams GD, Dick R, Zhang Z, Brewer GJ. Tetrathiomolybdate is effective in a mouse model of arthritis. J Rheumatol 2006;33:2501-2506.

25. Gelderman KA, Hultqvist M, Pizzolla A, et al. Macrophages suppress $\mathrm{T}$ cell responses and arthritis development in mice by producing reactive oxygen species. J Clin Invest 2007;117:3020-3028.

26. Park MK, Park JS, Cho ML, et al. Grape seed proanthocyanidin extract (GSPE) differentially regulates Foxp3 $(+)$ regulatory and IL-17(+) pathogenic $\mathrm{T}$ cell in autoimmune arthritis. Immunol Lett 2011;135:50-58.

27. Ahmad SF, Zoheir KM, Abdel-Hamied HE, et al. Grape seed proanthocyanidin extract has potent anti-arthritic effects on collagen-induced arthritis by modifying the $\mathrm{T}$ cell balance. Int Immunopharmacol 2013;17:79-87.

28. Medzhitov R, Preston-Hurlburt P, Janeway CA Jr. A hu- man homologue of the Drosophila Toll protein signals activation of adaptive immunity. Nature 1997;388:394-397.

29. Sadanaga A, Nakashima H, Akahoshi M, et al. Protection against autoimmune nephritis in MyD88-deficient MRL/ lpr mice. Arthritis Rheum 2007;56:1618-1628.

30. Kowalski ML, Wolska A, Grzegorczyk J, et al. Increased responsiveness to toll-like receptor 4 stimulation in peripheral blood mononuclear cells from patients with recent onset rheumatoid arthritis. Mediators Inflamm 2008;2008:132732.

31. Chovanova L, Vlcek M, Krskova K, et al. Increased production of IL-6 and IL-17 in lipopolysaccharide-stimulated peripheral mononuclears from patients with rheumatoid arthritis. Gen Physiol Biophys 2013;32:395-404.

32. Tang CH, Hsu CJ, Yang WH, Fong YC. Lipoteichoic acid enhances IL-6 production in human synovial fibroblasts via TLR2 receptor, PKCdelta and c-Src dependent pathways. Biochem Pharmacol 2010;79:1648-1657.

33. Lorenz W, Buhrmann C, Mobasheri A, Lueders C, Shakibaei M. Bacterial lipopolysaccharides form procollagen-endotoxin complexes that trigger cartilage inflammation and degeneration: implications for the development of rheumatoid arthritis. Arthritis Res Ther 2013;15:R111.

34. Radstake TR, Franke B, Hanssen S, et al. The Toll-like receptor 4 Asp299Gly functional variant is associated with decreased rheumatoid arthritis disease susceptibility but does not influence disease severity and/or outcome. Arthritis Rheum 2004;50:999-1001.

35. Yang H, Wei C, Li Q, et al. Association of TLR4 gene non-missense single nucleotide polymorphisms with rheumatoid arthritis in Chinese Han population. Rheumatol Int 2013;33:1283-1288.

36. Palsson-McDermott EM, O'Neill LA. Signal transduction by the lipopolysaccharide receptor, Toll-like receptor-4. Immunology 2004;113:153-162.

37. Bartok B, Firestein GS. Fibroblast-like synoviocytes: key effector cells in rheumatoid arthritis. Immunol Rev 2010;233:233-255.

38. Huang QQ, Pope RM. The role of toll-like receptors in rheumatoid arthritis. Curr Rheumatol Rep 2009;11:357364.

39. Davignon JL, Hayder M, Baron M, et al. Targeting monocytes/macrophages in the treatment of rheumatoid arthritis. Rheumatology (Oxford) 2013;52:590-598. 